\title{
Towards a new Class of Medical Biomarkers - Stable Calcium Isotopes, Osteoporosis and Beyond
}

\author{
A. EISENHAUER ${ }^{1 *}$, M. MÜLLER ${ }^{2}$, A. HEUSER ${ }^{1}$, A. \\ KOLEVICA ${ }^{1}$, C. LAUE $^{3}$, R. SHROFF ${ }^{4}$ AND J. \\ SCHREZENMEIR $^{3}$
}

${ }^{1}$ GEOMAR, Kiel, Germany

${ }^{2}$ University Medical Center (UKSH), Kiel, Germany

${ }^{3}$ Clinical Research Center Kiel (CRC) GmbH, Kiel, Germany

${ }^{4}$ Great Ormond Street Hospital, London, United Kingdom

A medical biomarker is defined as "any substance, structure, or process that can be measured in the body or its products and influence or predict the incidence of outcome or disease" [1]. A biomarker must be able to inform medical decisions, not be difficult, time consuming, or expensive that it cannot be done on a large number of samples. Following this approach isotopes of certain naturally occuring elements in the human body may have several advantages over traditional medical biomarkers and will probably introduce new, powerful, and practical sets of diagnostic tools. Among them stable Calcium (Ca) isotope ratios which may serve as sensitive biomarkers for osteoporosis and other diseases.

In a registered clinical study (NCT02967978, [2]) 100 postmenopausal women (50-75 years) underwent dual-energy Xray absorptiometry (DXA), the gold standard for determination of bone mineral density (BMD). After exclusion of women with kidney failure and vitamin D deficiency $(<25 \mathrm{nmol} / 1) 80$ women remained of which 14 fulfilled the DXA based criteria for osteoporosis (T-score<$2.5)$. Both the $\delta^{44 / 42}$ CaBlood $(\mathrm{p}<0.001)$ and $\delta^{44 / 42}$ CaUrine $(\mathrm{p}=0.004)$ values significantly differed between women with and without osteoporosis. $\delta^{44 / 42}$ CaBlood and $\delta^{44 / 42}$ Caurine correlated significantly ( $p=0.004$ to $p=0.031$ ) with DXA and also significantly with biomarkers (CRP, CTX/P1NP) associated with bone mineralization/demineralization. Cut-off values for discrimination of osteoporosis were $\sim-0.85 \%$ and $\sim 0.16 \%$ for $\delta^{44 / 42}$ CaBlood and $\delta^{44 / 42}$ Caurine, respectively. Corresponding sensitivities (specificities) were 100\% ( $\sim 55 \%)$ for $\delta^{44 / 42}$ CaBlood and $~ 79 \%(\sim 71 \%)$ for $\delta^{44 / 42}$ Caurine.

A post-hoc assessment of osteoporotic fractures in the following 2 years showed that DXA underestimates the fracture risk by a factor of two. Of the eight cases, 4 were predicted by DXA, 7 by the $\mathrm{Ca}$ biomarker in urine ( $\delta^{44 / 42}$ Caurine) and all 8 by the $\delta^{44 / 42}$ CaBlood measurements in blood serum.

[1] https://apps.who.int/iris/handle/10665/39037, [2] Eisenhauer et al. (2019) Bone Reports, 10. 